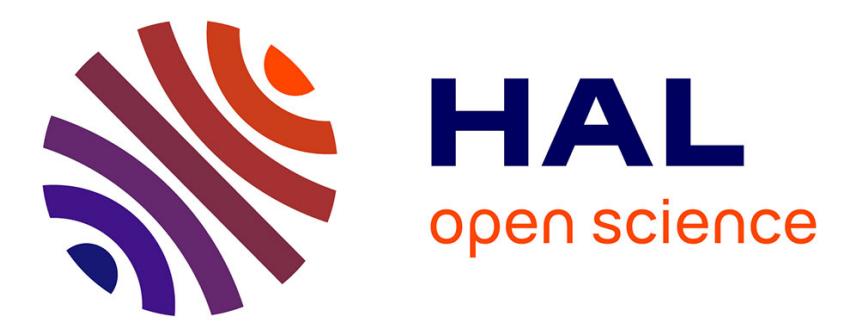

\title{
Développement d'un Système de Caractérisation des Agrégats et Flocs (SCAF)
}

Bernard Mercier, Valentin Wendling, Catherine Coulaud, Cédric Legout, Nicolas Gratiot

\section{- To cite this version:}

Bernard Mercier, Valentin Wendling, Catherine Coulaud, Cédric Legout, Nicolas Gratiot. Développement d'un Système de Caractérisation des Agrégats et Flocs (SCAF). 7ème Colloque Interdisciplinaire en Instrumentation, Jan 2016, Saint-Nazaire, France. hal-01280187

\section{HAL Id: hal-01280187 \\ https://hal.science/hal-01280187}

Submitted on 29 Feb 2016

HAL is a multi-disciplinary open access archive for the deposit and dissemination of scientific research documents, whether they are published or not. The documents may come from teaching and research institutions in France or abroad, or from public or private research centers.
L'archive ouverte pluridisciplinaire HAL, est destinée au dépôt et à la diffusion de documents scientifiques de niveau recherche, publiés ou non, émanant des établissements d'enseignement et de recherche français ou étrangers, des laboratoires publics ou privés. 


\title{
Développement d'un Système de Caractérisation des Agrégats et Flocs (SCAF)
}

Bernard Mercier ${ }^{1 *}$, Valentin Wendling ${ }^{1}$, Catherine Coulaud1, Cédric Legout ${ }^{1}$, Nicolas Gratiot ${ }^{1}$

\author{
1 Université Grenoble Alpes/CNRS/IRD, LTHE UMR 5564, 38041 Grenoble, France
}

\section{Résumé}

*auteur contact : bernard.mercier@ujf-grenoble.fr

L'étude du transport des matières en suspension dans les cours d'eau pour en déterminer les impacts revêt un intérêt grandissant. II n'existe cependant pas d'instrument capable d'effectuer des mesures de vitesse de sédimentation en temps réel et pour des régimes très concentrés, a fortiori en situation de crue. Pour répondre à ce besoin, un Système de Caractérisation des Agrégats et Flocs (SCAF) a été développé. L'appareil a été conçu pour s'intégrer dans un préleveur automatique, d'usage courant en hydrologie. L'une des difficultés principales a consisté à intégrer l'électronique de mesure aux flacons des préleveurs automatiques.

Lors de ses évaluations, le SCAF a permis des mesures dans des gammes de concentrations allant jusqu'à plusieurs dizaines de g/l, valeurs courantes pour des cours d'eau de montagne. Outre la détermination des vitesses de dépôt, la méthode de traitement des données permet de calculer un "indice de floculation" caractérisant l'aptitude des particules à s'agglomérer.

\begin{abstract}
Development of an "Aggregate and Floc Characterization System" (SCAF)

We know little regarding the processes that govern the evolution of suspended particle characteristics during their transport through a river basin. Up to now, no measurement method allows measuring continuously the suspended sediment properties in highly concentrated fluid flows, such as those observed in headwater catchments during runoff events or near bed in muddy estuaries. The Aggregate and Floc Characterization System (SCAF) has been developed in order to measure suspended sediment properties for a wide range of concentrations. It has been designed to be easily incorporated into automatic water samplers.
\end{abstract}

\section{Introduction}

Les cours d'eau transportent de nombreux matériaux d'origine minérale et biologique qui affectent les installations hydrauliques, les écosystèmes et la qualité de l'eau. Les matières en suspension (MES), constituées d'éléments dont la taille varie de quelques microns à quelques millimètres, ont la capacité à s'agréger au cours de leur transport pour constituer des "flocs" dont les propriétés physiques (taille, diamètre, vitesse de chute) diffèrent significativement de celles des particules élémentaires qui les constituent. Ce phénomène de "floculation" joue un rôle clé dans la dynamique sédimentaire avec un effet important sur les zones de dépôt. Une bonne connaissance des flocs est également essentielle pour des problématiques de qualité des milieux, les polluants pouvant être incorporés aux particules et ainsi être transportés et déposés avec elles. Pour comprendre et modéliser le devenir des sédiments et des polluants associés, les scientifiques, mais aussi les aménageurs ou les opérateurs d'installations hydrauliques souhaitent mesurer les vitesses de dépôt des particules. Des techniques de mesure in-situ de grandeurs caractérisant la vitesse de chute 
(taille, forme, densité) existent pour les zones côtières, fluviales et estuariennes [1]. Ces techniques ne sont toutefois pas transposables aux petits bassins versants de montagne, où les concentrations varient rapidement et peuvent fréquemment atteindre plusieurs dizaines de grammes par litre. Par ailleurs, l'étude de la floculation est difficile lors d'une mesure directe dans une rivière en crue sous l'effet de la turbulence. Pour répondre à ces besoins, un Système de Caractérisation des Agrégats et Flocs (SCAF) permettant de mesurer sur site des suspensions immédiatement après leur prélèvement a été conçu et développé au LTHE à Grenoble. Nous présentons ici ce développement.

\section{Principaux éléments du cahier des charges}

Le principe de l'instrument repose sur l'étude de la décantation des MES directement après prélèvement d'échantillons en mesurant l'évolution spatio-temporelle de la turbidité. Pour ce faire, des couples d'émetteurs/récepteurs de lumière sont répartis le long d'une colonne contenant la suspension. II s'agit de mesurer l'intensité de la lumière reçue par chaque capteur après interaction avec la solution et la variation de cette intensité selon la verticale au cours du temps. La mesure a lieu sur site pendant toute la durée de la décantation. A priori, une mesure en transmission (absorbance) ou en rétrodiffusion est possible, déterminée par les positions relatives des émetteurs et des détecteurs. Les signaux lumineux doivent être détectés dans des milieux à forte concentration en MES, typiquement quelques dizaines de grammes par litres.

Le second point important du cahier des charges est de placer le système de mesure dans un préleveur automatique, fréquemment employé dans les stations de suivi hydrosédimentaire. En effet, de nombreux scientifiques et opérateurs possèdent déjà de tels appareils pour prélever des échantillons d'eau en rivière ou en lac puis déterminer en laboratoire les concentrations de MES. Ainsi, il sera possible à ces utilisateurs, sur la base de l'existant, d'accéder également et si possible à moindre coût, à la mesure de vitesse de dépôt effectuée immédiatement après le prélèvement.

Les préleveurs automatiques sont équipés de flacons en plastique (24 sur notre modèle) d'une contenance d'environ 1 litre, disposés en corolle. Les échantillons sont recueillis dans les flacons au moyen d'une douchette d'injection décrivant un cercle pour passer d'un flacon à l'autre en fin de remplissage. L'idée est d'incorporer un dispositif de mesure dans chacun des flacons que l'on souhaite équiper. Cette demande du cahier des charges constitue une contrainte en termes de miniaturisation de l'électronique et d'étanchéité. II est possible de mixer un nombre quelconque de flacons instrumentés avec le complément en flacons d'origine. Dans le contexte d'un dispositif prototype, il a été décidé de ne pas modifier mécaniquement l'appareil commercial de prélèvement ni d'intervenir sur ses fonctions électroniques et logicielles initiales.

\section{Description du système de mesure}

Les principes de mesure et la configuration générale étant donnés, les principales questions qui se posent sont les suivantes :

1- Comment intégrer l'électronique de mesure d'un dispositif dans le flacon d'origine? 
2- Un système de pilotage et acquisition étant requis, celui-ci doit-il être centralisé ou propre à chaque dispositif de mesure?

3- Doit-on travailler en transmission ou en rétrodiffusion?

Ces questions interagissent et la réponse à chacune d'elle a des implications sur celles apportées aux autres.

\subsection{Choix de l'architecture du système}

Concernant le premier point, l'électronique du système de mesure étant incluse dans le flacon qui fait office de boitier, il n'est plus possible de remplir le flacon directement. La solution qui a été retenue consiste à utiliser un récipient intermédiaire logeable facilement dans le flacon. Le choix s'est porté sur un tube en verre cylindrique à fond plat d'un diamètre extérieur égal à l'embouchure du flacon et d'une hauteur telle qu'il affleure celle-ci (respectivement $40 \mathrm{~mm}$ et $24 \mathrm{~cm}$ sur notre modèle). Le tube est en verre pour une transmission optimale et durable du signal lumineux (possibilité de lavage après extraction). Du point de vue du phénomène physique étudié, la réduction du volume recueilli peut altérer la dynamique de décantation lorsqu'on a affaire à des particules cohésives. Ceci ne semble pas être observé avec un diamètre intérieur de tube de $35 \mathrm{~mm}$.

Un support mécanique a été conçu pour y fixer les cartes électroniques et maintenir le tube. Le flacon a été modifié pour insérer l'ensemble : le fond a été découpé et la base du support constitue le nouveau fond du flacon. Un joint plat étanchéifie cette nouvelle base. Un second joint, torique, placé au niveau de l'embouchure réalise l'étanchéité au sommet du tube. Un connecteur étanche est placé sur la surface supérieure du flacon pour permettre l'alimentation électrique et la communication des données.

La réponse à la seconde question est plus simple. D'une part, le nombre de connexions à réaliser vers un système de pilotage et acquisition centralisé serait prohibitif. D'autre part, le niveau d'intégration et le coût réduit des composants permettent aujourd'hui d'implanter cette fonction sur chaque dispositif unitaire. La solution mécanique retenue laisse un espace suffisant pour la loger. En contrepartie, il est nécessaire d'ajouter au système une petite fonction commune pour la configuration des dispositifs de mesure et le recueil des données, gérant un lien de communication avec ceux-ci.

La troisième question a été traitée lors de l'étude de faisabilité réalisée hors contexte d'intégration dans un préleveur. Un support autonome réalisé à cette occasion permettait de modifier la position angulaire d'une barrette de récepteurs par rapport à une barrette émettrice relativement à l'axe du tube. Les modes transmission et rétrodiffusion ont ainsi été testés. C'est finalement le mode transmission, c'est à dire la mesure de l'absorbance de la lumière par les MES, qui a donné les meilleurs résultats. Ceci est principalement dû au fait que, le système ayant été conçu pour étudier les solutions à hautes concentrations, les mesures par rétrodiffusion arrivent plus rapidement à saturation (Wendling et al., 2015).

\subsection{Description fonctionnelle d'un dispositif de mesure}

Un dispositif de mesure unitaire comprend 3 fonctions: émission, réception et commande-acquisition, qui vont être présentées. Dans toute cette section, on se référera à la figure 1. 


\subsubsection{Fonctions émission/réception}

La mesure s'effectuant par absorption du signal lumineux, deux cartes séparées sont nécessaires. La première est équipée d'émetteurs de lumière et la seconde de photodétecteurs. Les couples émetteurs/récepteurs sont disposés en vis-à-vis selon un diamètre du tube recueil. Ces cartes sont disposées verticalement et affleurent la paroi externe du tube. Les composants optoélectroniques sont à montage de surface (CMS) inverse, c'est à dire soudés sur la face arrière des cartes et dirigés vers la surface du tube au travers d'un perçage des circuits imprimés, de sorte que leur partie sensible soit au plus près de cette surface.

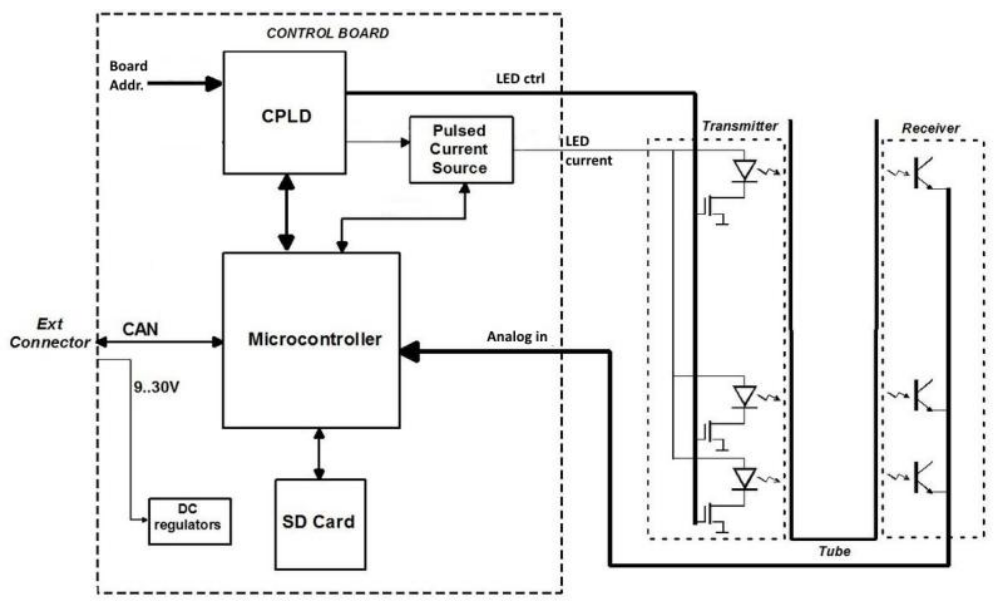

Figure 1 : Diagramme du système électronique de mesure.

Une résolution spatiale de $10 \mathrm{~mm}$ a été jugée réaliste, ce qui, compte tenu de la hauteur des flacons et des convenances techniques a conduit au choix de l'utilisation de 16 couples de composants optoélectroniques. Le premier couple étant également placé à $10 \mathrm{~mm}$ du fond du tube, la hauteur d'analyse de chute est de $16 \mathrm{~cm}$.

Les émetteurs sont des diodes électroluminescentes (LED) à haute intensité lumineuse $(40 \mathrm{~mW} / \mathrm{sr})$ et faible angle d'émission $\left( \pm 12^{\circ}\right)$, dont le rayonnement est centré sur $890 \mathrm{~nm}$. Les détecteurs sont des phototransistors dont la bande passante va de 730 à $1000 \mathrm{~nm}$, donc sensibles à cette longueur d'onde mais insensibles aux longueurs d'onde visibles, ce qui améliore le rapport signal à bruit de la mesure.

Chaque LED est dotée d'un dispositif de commutation (transistor MOS) qui leur permet d'être allumées en séquence. On élimine ainsi toute ambigüité sur l'origine du signal lumineux détecté à un instant donné. De plus, cela permet de respecter les valeurs maximales (données par le constructeur) de durée d'éclairement et de période de répétition des impulsions pour le courant le plus fort sous lequel les LED sont amenées à être alimentées. Comme le montre la figure 2, il existe un temps d'extinction totale entre les périodes d'émission de deux LED consécutives, qui sera mis à profit lors de la mesure. Dans notre cas, la durée d'éclairement d'une LED est de $100 \mu \mathrm{s}$, le temps séparant les allumages de 2 diodes successives est de $625 \mu \mathrm{s}$, soit un balayage de la barrette complète en $10 \mathrm{~ms}$.

L'alimentation des LED est commune et s'effectue en courant. 


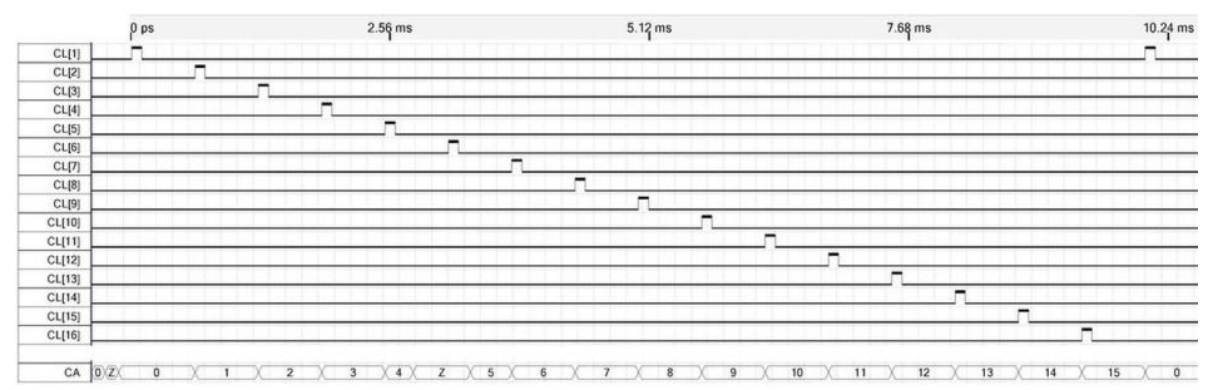

Figure 2 : Séquence d'allumage des LED.

Chaque phototransistor est suivi d'un amplificateur adaptant le signal à l'entrée des convertisseurs analogiques/numériques utilisés pour l'acquisition.

\subsubsection{Partie pilotage-acquisition}

C'est la troisième carte du dispositif. Ses quatre fonctions essentielles sont :

- la génération des signaux de commande des commutateurs de LED ;

- I'acquisition des signaux analogiques de mesure en les convertissant en signaux numériques ;

- le stockage des valeurs dans une mémoire électronique sous forme de fichiers de données, après prétraitement des signaux de mesure numérisés.

- la communication avec l'extérieur pour paramétrer le dispositif ou envoyer les fichiers de mesure.

Cette carte accueille aussi les fonctions régulateurs de tension et alimentation en courant des LED.

La présence de fonctions telles que le stockage de données en mémoire et de communication implique l'utilisation d'un microcontrôleur. II est apparu judicieux de choisir un modèle intégrant un convertisseur analogique-numérique et un multiplexeur doté de 16 entrées. Cette fonctionnalité n'est pas si courante mais elle a pu être satisfaite avec un microcontrôleur de la famille STM32F1 qui dispose des 16 entrées analogiques et d'un convertisseur 12 bits. II dispose par ailleurs de toutes les autres fonctions nécessaires, comme la mémorisation des données dans une carte micro-SD via un bus SPI. La donnée de mesure de chaque étage est obtenue à chaque pas de temps par différence des valeurs des signaux lumineux reçus pendant la période d'allumage et pendant la période d'extinction pour s'affranchir d'éventuels signaux parasites. L'intégralité des données au pas de temps de $10 \mathrm{~ms}$ est mémorisée pendant les 2 premières minutes pour prendre en compte une chute rapide de matière non cohésive. Une moyenne sur un nombre d'échantillons et à un pas de temps tous deux programmables est effectuée ensuite.

Le pilotage des commutateurs de LED est assuré par un circuit logique programmable (CPLD), pour ne pas avoir à solliciter trop de broches d'entrées/sorties du microcontrôleur ni surcharger son programme. On assure ainsi une régularité absolue des temps. Un mécanisme de synchronisation est en place entre les deux circuits.

Concernant la communication vers l'extérieur, on a opté pour le bus CAN, bus série à deux fils parfaitement capable de gérer les 24 flacons instrumentés. Le microcontrôleur choisi 
possède une interface pour ce bus. Une adresse est affectée à chaque carte, permettant de reconnaître le flacon. L'alimentation est commune à tous les dispositifs unitaires et fournie depuis l'extérieur de ces dispositifs. Cela peut notamment être celle utilisée pour le préleveur automatique (batterie12 ou 24V). La puissance consommée par flacon est de $1 \mathrm{~W}$.

La figure 3 donne un aperçu de la réalisation, avec les trois cartes montées sur le support interne. La liaison des deux cartes optoélectroniques avec la carte de commande s'effectue par nappe flexible.
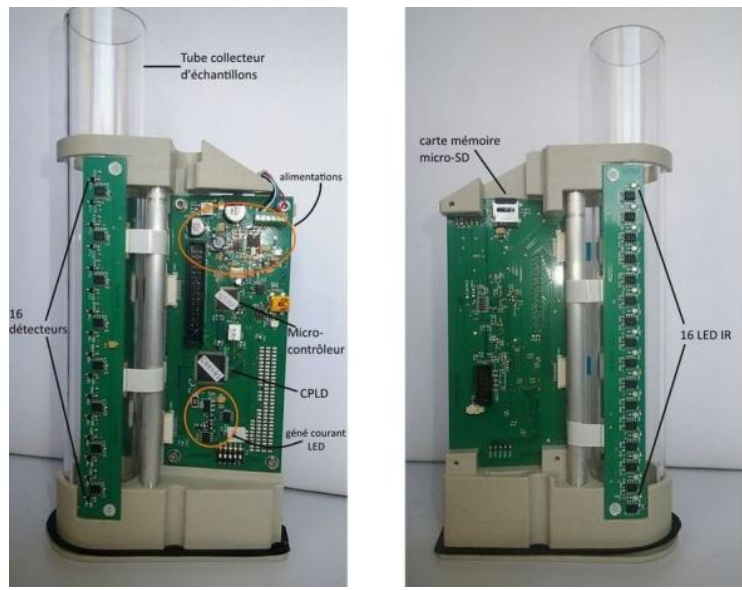

Figure 3 : Cartes électroniques sur support a) vue avant b) vue arrière.

L'appareil complet nécessite un dispositif supplémentaire. Ce dispositif commun a une fonction de contrôleur de bus CAN et d'interface USB pour la connexion à un ordinateur. II peut être présent en permanence ou seulement lors des phases de paramétrage des dispositifs et de récupération des données (figure 4b).
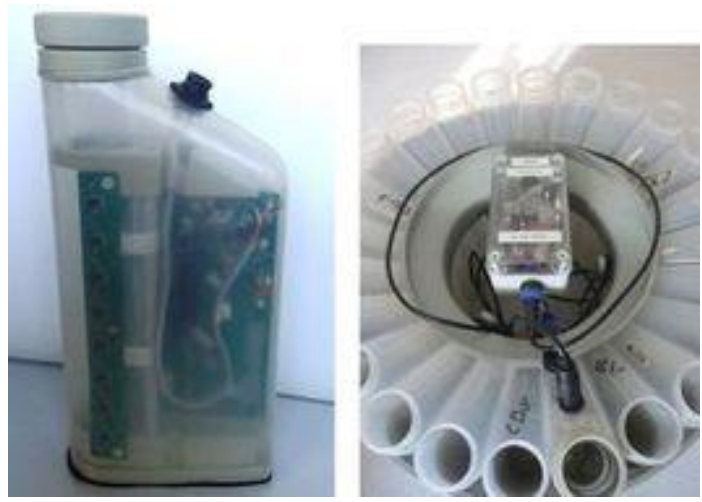

Figure 4 : a) Flacon instrumenté b) flacon dans le préleveur, bus et contrôleur CAN/interface USB. 


\section{Traitement des données}

Le système fournit les variations temporelles de l'éclairement reçu par les 16 photodétecteurs sous la forme de valeurs de tension. Ces données sont alors traitées pour obtenir des courbes d'iso-absorbance en fonction du temps. La méthode appliquée aux isoconcentrations présentée dans [2] a été étendue aux iso-absorbances pour déterminer la distribution des vitesses de chute [3]. Pour des matériaux non cohésifs tels les sables, les particules chutent à vitesse constante et les courbes d'iso-absorbance sont linéaires (Figure 5-a). Lorsque les particules s'agrègent pendant leur dépôt, leur vitesse augmente au cours du temps (Figure 5-b). Le rapport entre les vitesses au fond du tube et celles à la surface fournit un indice caractérisant la propension du matériau à floculer, variant de 0 pour des matériaux non cohésifs (sable) pour atteindre plus de 50 pour des sédiments fin naturels.

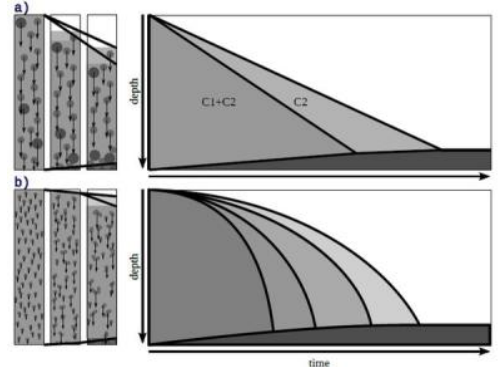

Figure 5 : Courbes d'iso-absorbance pour (a) des matériaux non cohésifs en suspension et (b) des matériaux qui floculent au cours de leur sédimentation.

Lors de son utilisation, le SCAF a montré sa capacité à traiter des solutions dans des gammes de concentrations allant de quelques dizaines de milligrammes par litre à plusieurs dizaines de grammes par litre, ce qui répond au besoin initial. Au-delà d'une dizaine de grammes par litre, le faisceau lumineux n'est plus détecté, et c'est la descente de l'interface eau claire-sédiments qui est suivie. Les essais ont toutefois montré la nécessité de prendre en compte la dispersion des caractéristiques des couples optoélectroniques. Ceci est actuellement réalisé par une inter-calibration préalable des capteurs.

\section{Conclusion et perspectives}

Un Système de Caractérisation des Agrégats et Flocs a été développé pour permettre de mieux caractériser les propriétés physiques des matières en suspensions, notamment dans les bassins versants de montagne.

Des prototypes autonomes non intégrés en station de suivi hydro-sédimentaire ont été réalisés en plusieurs exemplaires. Ils sont utilisés par des chercheurs et des ingénieurs (EDF) pour caractériser la dynamique des rivières turbides lors de campagnes de terrain, généralement suivies d'essais complémentaires en laboratoire. Une méthode de traitement et d'analyse des données a été développée au cours d'un travail de thèse (Wendling, 2015). Les mesures ont montré la capacité de l'appareil à analyser des solutions dans de larges 
gammes de concentration. Certaines limitations inhérentes à la phase de développement demeurent et font l'objet de nos efforts.

Forts de ces résultats, la version intégrée dans un préleveur automatique a été développée. Son fonctionnement, autonome, sur le terrain fera l'objet des prochaines validations.

A cette étape du développement, le SCAF démontre un grand potentiel pour une caractérisation des propriétés physiques des particules dans des conditions hydrosédimentaires extrêmes. L'intérêt des scientifiques pour ces nouvelles mesures nous incitent maintenant à rechercher des coopérations en vue d'un transfert technologique.

\section{Remerciements}

Ce dispositif a bénéficié de financements de I'INSU (EC2CO BIOHEFECT), de l'ANR dans le cadre d'un projet Jeunes Chercheurs (ANR-12-JS06-0006) et du soutien d'EDF.

\section{Bibliographie}

[1] Mantovanelli, A., Ridd, P. V. "Devices to measure settling velocities of cohesive sediment aggregates: A review of the in situ technology". Journal of Sea Research. Vol. 56. no. 3, p.199226 (2006)

[2] Piro, P., Carbone, M., Penna N., Marsalek, J. "Characterization of the settling process for wastewater from a combined sewer system". Water Research. Vol. 45, $n^{\circ} 20$, p.6615-6624 (2011)

[3] Wendling, W., Gratiot, N., Legout, C., Droppo, I. G., Coulaud, C., Mercier, B. « Using an optical settling column to assess suspension characteristics within the free, flocculation, and hindered settling regimes », J. of Soils \& Sediments, Vol. 15, Issue 9, p. 1991-2003 (2015) 